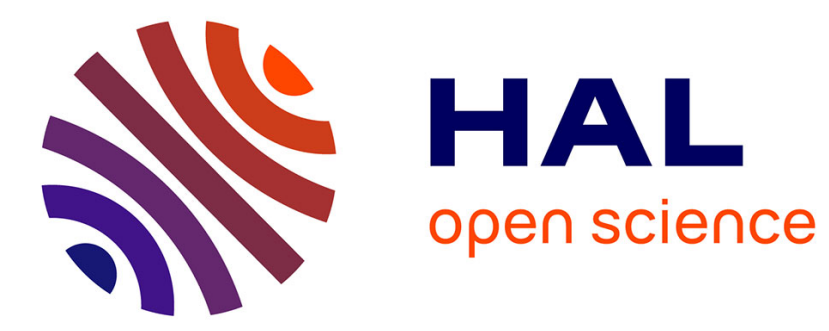

\title{
Dynamics of spatial structure in French urban agglomerations
}

Denise Pumain, Thérèse Saint-Julien, Lena Sanders

\section{To cite this version:}

Denise Pumain, Thérèse Saint-Julien, Lena Sanders. Dynamics of spatial structure in French urban agglomerations. Papers of the Regional Science Association, 1984, 55, pp.71-82. halshs-01488316

\section{HAL Id: halshs-01488316 https://shs.hal.science/halshs-01488316}

Submitted on 13 Mar 2017

HAL is a multi-disciplinary open access archive for the deposit and dissemination of scientific research documents, whether they are published or not. The documents may come from teaching and research institutions in France or abroad, or from public or private research centers.
L'archive ouverte pluridisciplinaire HAL, est destinée au dépôt et à la diffusion de documents scientifiques de niveau recherche, publiés ou non, émanant des établissements d'enseignement et de recherche français ou étrangers, des laboratoires publics ou privés. 
DYNAMICS OF SPATIAL STRUCTURE IN FRENCH URBAN AGGLOMERATIONS

D. PUMAin(1) T. SAINT JULien(2) L. SANDERS(2)

(1) I.N.E.D., 27 rue du Commandeur, 75014 Paris, France

(2) Centre de Géographie Théorique, 13 rue du Four, 75006 Paris

\section{ABSTRACT}

P.ALLEN's INTRAURBAN model applies bifurcation theory to simulate the evolution of urban spatial structure. Until now, this model has only been tested to simulate plausible fictitious urban structures but its ability to reproduce observed evolutions has still to be tested. We carried out such an application to post-war evolution of spatial structure in a few french urban agglomerations. Many problems were encountered in choosing the data, estimating parameters and calibrating the model. The high complexity of the model raises up much theoretical interest but makes calibration rather difficult.

The INTRA URBAN model was drawn up by researchers of the "Brussel's school" in systems dynamic [1] [2]. It is one of the first models of bifurcation-type designed to describe the dynamics of spatial systems. That kind of model use equations which were first established to simulate auto-organization phenomena in open physical systems situated far from equilibrium. These models allow two kinds of changes in the system : progressive evolution along deterministic trajectories defined by non-linear equations; and sudden bifurcations, or change in trajectories, which may be produced by random fluctuations. These bifurcations alter the structure of the system. Such a conception of change is rather attractive for social sciences [3] and seems to open new ways to the study of urban dynamics.

Inded, two series of models already designed and presented by P. Allen 
have proved their ability to reproduce theoretical and plausible long-range or medium-range evolutions of fictitious spatial systems : on the one hand by simulating the development of a central places network [4]; and on the other hand by generating various internal configurations of economic activities and residences in a large metropolitan area [2].

Before recording particular problems encountered and preliminary results in our tentative applications, we shall first recall the main specificities and mathematical structure of the INTRA URBAN model.

\section{I - A DYNAMIC MODEL FOR INTRA-URBAN SPATIAL STRUCTURES}

One characteristic of INTRA-URBAN model, of particular interest for geographers, is that it is basically a dynamic and spatial model, which is a great advantage comparing for example to FORRESTER's urban dynamics model. In ALLEN's model, urban space is considered as an open system, made up of about twenty parts (urban districts or communes). Each town-part is competing with each other for attracting jobs and residents. Each town-part is characterized by :

- a number of jobs in different economic activities (industry, exporting tertiary, short-range and medium-range tertiary activities) ;

- a number of resident population having different occupational status (blue collars and white collars);

- a location respective to other town-parts and accessibility indices to transportation and information networks.

Starting from a given initial configuration, the model calculates variations in the number of jobs and residents of each kind in each townpart, according to spatial and non-spatial interactions between them. 
Contrary to its apparent over-simplification of urban structure, the model recaptures the high complexity of urban dynamics by defining these interactions. They are designed as mathematical relationships between variables or parameters standing for empirical regularities, theoretical hypothesis or conceptual entities often encountered in the litterature about spatial dynamics, but not having yet been put all together in a single model. INTRA URBAN model thus incorporates :

- a logistic type model of growth for jobs and population of each kind in every town-part ;

- the principles of economic base theory, dividing jobs between exporting activities (the growth of which depends upon an external demand) and nonexporting activities (the growth of which depends upon local demand, by means of induction rates) ;

- a distance-decreasing-interaction model, of the exponential type with intervening opportunities, for people working in a town-part and choosing a residence location ;

- some principles of location theory for economic activities, including an "internal cooperativity" parameter standing for externalities and similarities in locational preferencies of each activity sector, and a "sensitivity to crowding" parameter figuring unequal needs in floorspace according to the nature of economic activity, competition for space, and saturation effects ;

- The model also incorporates hypothesis about actor behaviour in an urban context, for example grouping and segregative tendencies inside and between social groups ; information theory is requested to introduce some delay in reaction of entrepreneurs or individuals to a potential demand 
and to define a parameter quoting for unequal information diffusion about quality of places among urban actors.

II - DESCRIPTION OF THE EQUATIONS

Each equation describes the variation, per unit of time, of the number of jobs or residents in a town-part $j$. Each equation takes the following form :

$$
\frac{d y j}{d t}=a y j(K-y j)
$$

where y $j$ figures the number of jobs or residents already located in zone $j$, a (settled for $\epsilon$ or $\eta$ ) is a speed of reaction to offer or to demand, and $K$ is a potential demand which determines the magnitude of the variation. (See tables 1 and 2 for the significance of parameters). This variation is then first a function of preexisting quantities of jobs or residents in considered town-part, which are growing according to a logistic curve.

1 - For activities depending upon an external demand (equation (1), industry and exporting tertiary), this variation is also a function :

- of external demand D;

- of relative attractivity of each zone $j$ for this kind of activity ; it is a ratio between intrinsec attractivity of zone $j$ and the sum of all attractivities of zones $j$. The attractivity of a zone $j$ is proportional to:

- the volume of jobs of the same kind already present in that zone ;

- the internal cooperativity of the activity being considered ( $\rho$ parameter) ;

- the sensitivity to crowding $(\tau)$;

and inversely proportional to :

- production cost $\mu$ 
Table 1 : Equations of INTRA-URBAN models.

1 - Exporting activities

$$
\begin{aligned}
& \frac{\mathrm{dS}^{E}}{\mathrm{dt}}=\epsilon^{E} S_{j}^{E}\left(D^{E} \frac{A_{j}}{\sum A_{j}^{\prime}}-S_{j}^{E}\right) \\
& \text { with } \quad A j=\left[\frac{\left(1+e^{E} S_{j}^{E}\right)}{\mu^{E}+\alpha_{j} \phi^{E}} \cdot \frac{\tau^{E}}{\tau^{E}+\sum_{k^{\prime}} x_{j}^{k^{\prime}}+\sum_{\ell^{\prime}} S_{j}^{\ell^{\prime}}}\right]^{C_{0}^{E}}
\end{aligned}
$$

$S_{j}^{E}=$ number of employments of activity $E$ in zone $j$

$$
\begin{aligned}
& \mathrm{E}=1=\text { industry } \\
& \mathrm{E}=2 \text { = exporting tertiary }
\end{aligned}
$$

2 - Short-range and medium-range tertiary functions

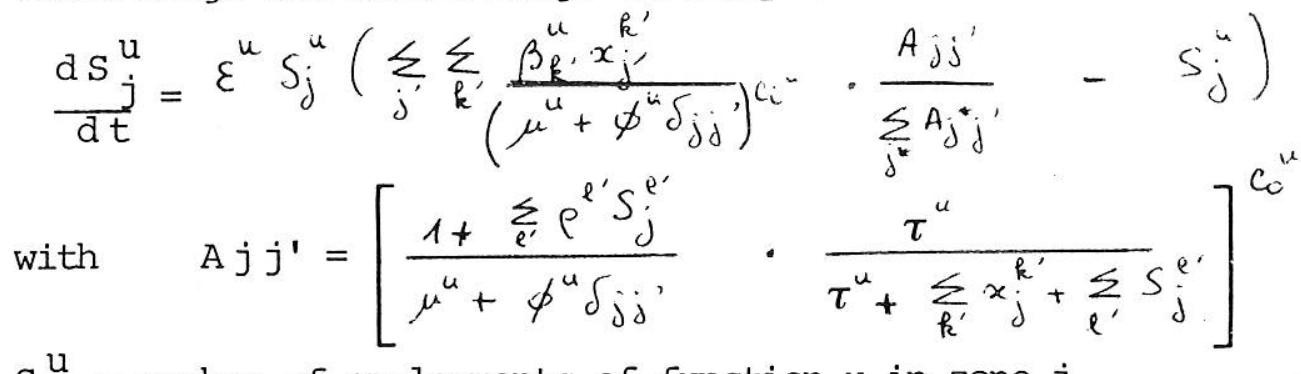

$\mathrm{s}_{j}^{\mathrm{u}}=$ number of employments of function $u$ in zone $j$

$\mathrm{u}=3$ = short-range function (local tertiary)

$\mathrm{u}=4$ = medium-range function (regional tertiary)

3 - Resident population

$$
d x_{j}^{k}=\eta^{k} x_{j}^{k}\left[\sum_{j^{\prime}}\left(\sum_{l^{\prime}} \mathrm{z}^{k l^{\prime}} S_{j}^{e^{\prime}} \frac{R_{j j}{ }^{\prime}}{\sum_{j^{*}} R_{j^{*} j^{\prime}}}\right)-x_{j}^{k}\right]
$$

with

$$
R j j^{\prime}=\left[\frac{v^{k}\left(1+\sigma^{k} x_{j}^{j^{k}}\right)}{e^{-b^{k} \delta_{j j^{\prime}}}}\right]
$$

$x_{j}^{k}=$ number of residents of category $k$ in zone $j$

$\mathrm{k}=\mathrm{l}=$ blue collars

$\mathrm{k}=2$ = white collars

$j j^{\prime}=$ distance between $j$ and $j^{\prime}$

for the significance of parameters, see table 2 . 
Table 2 : Significance of parameters

\begin{tabular}{|c|c|c|}
\hline Symbol & Significance & $\begin{array}{l}\text { Values in } \\
\text { first simulation }\end{array}$ \\
\hline$\alpha(17,2)$ & $\begin{array}{l}\text { For each zone, accessibility index to } \\
\text { transportation and information network }\end{array}$ & .1 to \\
\hline $\mathrm{b} \quad(2)$ & $\begin{array}{l}\text { Distance-decay parameter for each resident } \\
\text { population category }\end{array}$ & .3 \\
\hline$\beta(2,2)$ & $\begin{array}{l}\text { Induction rate of short range and medium } \\
\text { range tertiary function by residents of } \\
\text { each category }\end{array}$ & $.5 \cdot 3$ \\
\hline $\mathrm{Ci}$ (2) & $\begin{array}{l}\text { Elasticity of demand for the cost of each } \\
\text { service }\end{array}$ & 2. \\
\hline Co $(4)$ & Information available to the actors & $10-10-10-10$ \\
\hline$\epsilon(4)$ & $\begin{array}{l}\text { Speed of reaction of entrepreneurs to the } \\
\text { demand }\end{array}$ & $.001 \quad .01 \quad .01$ \\
\hline$\eta(2)$ & $\begin{array}{l}\text { Speed of adjustment of resident population } \\
\text { to employment offer }\end{array}$ & .005 \\
\hline$\varnothing(4)$ & Transportation cost for each activity & $.02 \quad .0001 \quad .025 \quad .005$ \\
\hline$\mu(4)$ & Production cost for each activity & $\begin{array}{llll}1 & 1 & 1 & 1\end{array}$ \\
\hline$v(2)$ & Sensitivity to crowding for residents & 1000 \\
\hline$\rho(4)$ & $\begin{array}{l}\text { Internal cooperativity for each activity } \\
\text { sector }\end{array}$ & $.01 \quad .01 \quad .0011 \quad .0011$ \\
\hline$\sigma(2)$ & $\begin{array}{l}\text { Grouping tendency for a category of } \\
\text { residents }\end{array}$ & .2 \\
\hline$\tau(4)$ & Sensitivity to crowding for each activity & $1000-3000-1000-2000$ \\
\hline $\mathrm{Z}(2,4)$ & $\begin{array}{l}\text { Share of each category of residents working } \\
\text { in each activity sector }\end{array}$ & 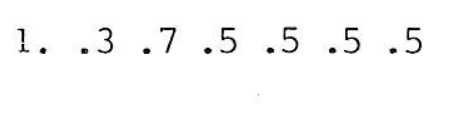 \\
\hline $\mathrm{D}(2)$ & External demand & $500-350$ \\
\hline
\end{tabular}


- transportation cost $\varnothing$ weighted by accessibility index of zone $j$ to transportation network $\alpha$ j ;

- saturation of space in town-part being considered, as measured by total number of jobs and residents in that zone.

Each attractivity is exponentiated by the mean of a $\mathrm{CO}$ parameter which figures the level of unanimity in territorial preferences among urban actors.

2 - For short-range and medium-range tertiary activities (equation (2), local and regional services), employment variation is a function of internal demand emanating from each zone.

Internal demand for a type of services (function $u$ ) in a zone $j$ ' is proportional to the total number of resident population, weighted by the mean level of demand per inhabitant for this kind of activity (induction rate $\beta$ ), and inversely proportional to the cost of this service. Cost is affected with an exponent $\mathrm{Ci}$ which figures degree of elasticity of services demand according to their cost. Cost incorporates production cost $\mu$ and transportation cost $\phi$, weighted by the distance $\delta j j '$ between place of production $j$ and place $j$ ' where consumers are living. This potential demand for services from zone $j$ ' to zone $j$ is weighted by relative attractivity of $z o-$ ne $j$ for zone $j$ ' (it is the ratio between attractivity of $j$ for $j$ ' and sum of attractivities for $j$ ' from all town-parts).

Attractivity of a zone $j$ for a zone $j$ ' is proportional to :

- the volume of preexisting jobs of type $u$ in $j$;

- the internal cooperativity of function $u(\rho)$;

- the intensity of the crowding supported by the function $u$; 
and inversely proportional to :

- production cost $\mu$;

- transportation cost $\varnothing$ weighted by the distance $\delta j j$ ' between zone $j$ and zone $j^{\prime}$;

- crowding in zone $j$ as measured by total number of employments and residents already located at $j$.

Each attractivity is exponentiated by the mean of a Co parameter standing for degree of uniformity in territorial preferences among the actors.

3 - For the two categories of residents (equation (3), blue collars and white collars), variation in a zone $j$ is function of the number of residents of each category employed by the four economic activities in every other town-part $j$ ', weighted by residential attractivity $R j j$ ' of zone $j$ for people working in zones $j^{\prime}$. Residential attractivity is a ratio between intrinsic attractivity of zone $j$ upon labor force working in $j$ ' and the sum of residential attractivity of all town-parts.

Residential attractivity of a zone $j$ for a category of people working in a zone $j^{\prime}$ is proportional to :

- number of residents of this category already present in $j$;

- affinity between members of a population of the same type $\sigma$ ) ;

- intensity of crowding supported by this category of residents $v$.

Residential attractivity decreases :

- proportionally with spatial crowding as measured by total number of employment and residents in $j$; 
- exponentially with distance between residential place $j$ and working place $j^{\prime}$, according to a gradient $b$ which measures sensitivity of each category to distant commuting.

The program realizes integration of each variable by an iterative procedure. It is possible to choose the time-lag for calculation and duration of the simulation. Finally, the program allows for introduction, at various stages of the simulation, of random fluctuations in the variable values.

\section{III - APPLICATION}

For the first test of the model, we choosed the urban agglomeration of Rouen. It offered sufficient similarities with the fictitious metropolitan area used by P. Allen for theoretical simulations : a large urban area ( 400.000 inhabitants), divided between 17 communes, the commune of Rouen being in a central position and being more than ten times larger by residents and employments number than other communes of the agglomeration. Heavy industry is mainly located, as in the theoretical case, along a river, the Seine.

\section{1 - The data}

For each of the 17 communes, we collected comparable data for 1954 , 1962, 1968 and 1975 (dates of censuses). Labor force is registred at workplace and had to be divided between four types of economic activity. Existing nomenclature allows only direct calculation for number of people employed in industry. To distinguish three levels (local, regional and exporting) among tertiary activities, we applied the minimum requirement technique. The two categories of resident population also stemmed from census 
data (socio-professional nomenclature), by grouping all workers as "blue collars" and all others as "white collars".

Relative location of the 17 communes are figured by geographic coordinates of their centre of gravity. Their accessibility index for communication lines needed by industry was quantified by separating communes situated along the Seine river $(\alpha(j, 1)=.5)$ and others $(\alpha(j, 1=1$.$) .$ As for information network, only the commune of Rouen was given a more favorable situation $(\alpha(j, 2)=.1)$ than the others $(\alpha(j, 2)=1$.$) .$

\section{2 - First simulation : american-type evolution}

In order to specify how the Rouen's evolution differed from some american-like theoretical cases studied by Allen et al. [2], we made a simulation using the same set of parameters (values in table 2). Results are plotted on figure 1 for the commune of Rouen (Center), all other communes being aggregated (Periphery), with dashed lines representing observed evolutions, for each of the six variables. These graphics show the following tendencies :

- a very rapid decline of industrial employment in the agglomeration center and a stagnation in periphery (in fact, industry stagnated between 1954 and 1975 in Rouen and growed slowly at the periphery, a little more in a few communes situated along the river) ;

- a slight move of concentration of exporting tertiary in the center (on the contrary, Rouen lost employments mainly to the benefit of two or three communes situated on the right bank) ;

- a very large growth of the number of local tertiary employments, which are developing quicker in the periphery than in the center (this is almost the observed evolution) ; 


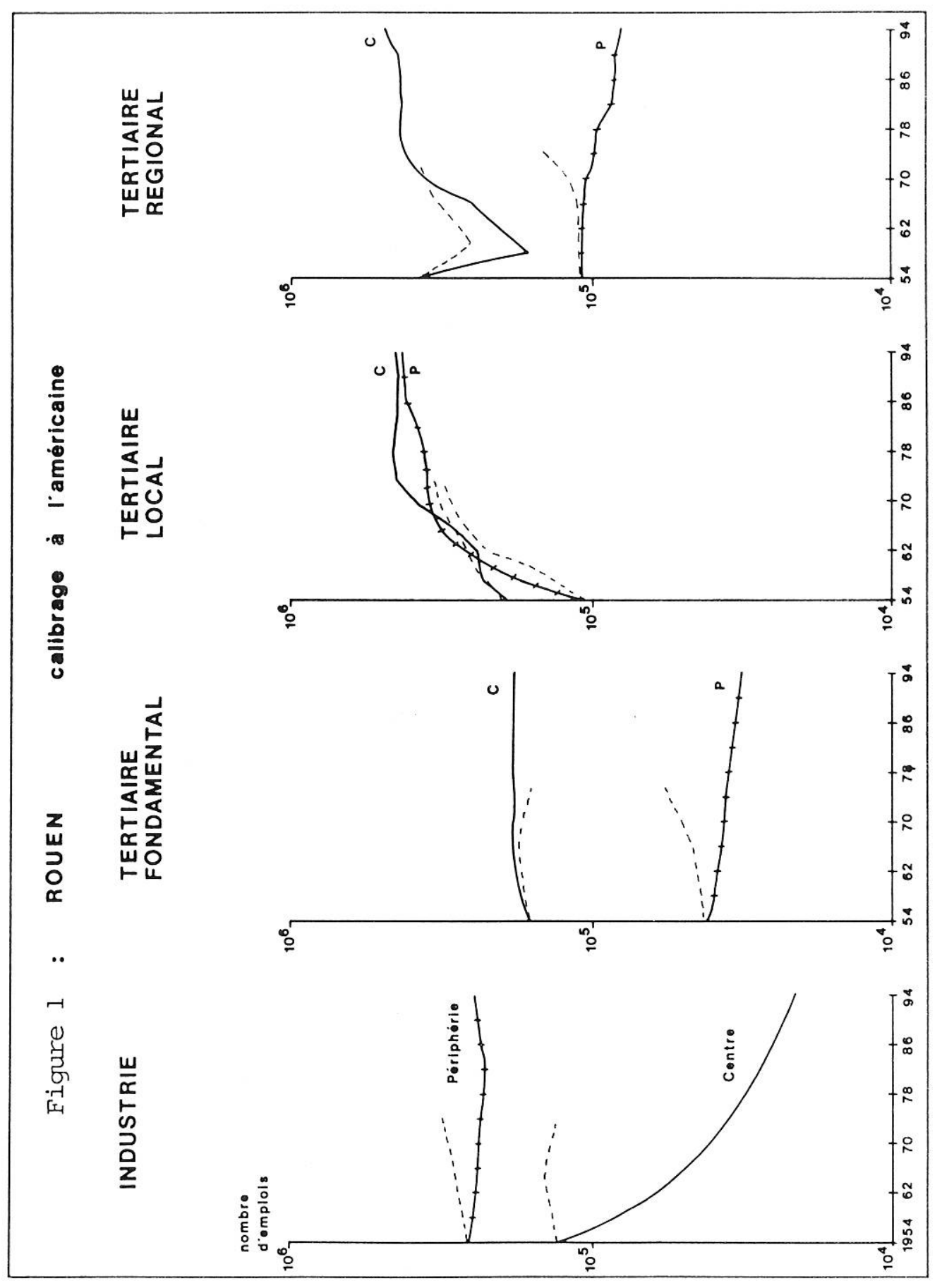




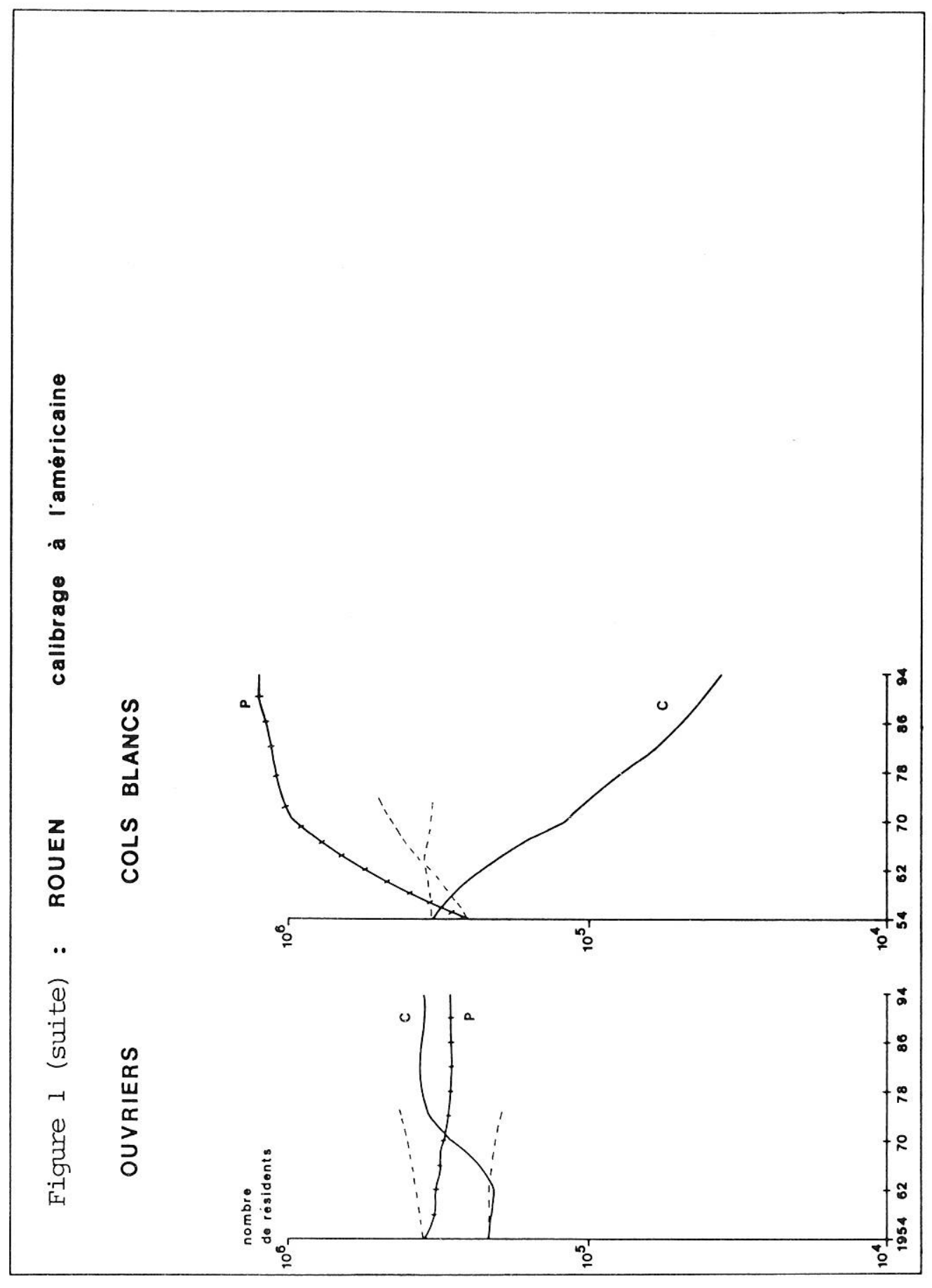


- after a quick departure from the center, regional tertiary employment concentrates again on that commune, while stagnating at the periphery (the observed evolution carries on the first tendency) ;

- blue collars are concentrating heavily in the center of the agglomeration (the observed evolution is almost the opposite) ;

- white collars leave completely the center and disperse in the whole periphery, more and more farthest (actually, the number of white collars was growing faster in the periphery but not leaving the centre of the agglomeration) .

This first trial shows strong differencies between simulated and observed evolution. In order to fit results to observed data, we had to change the values of the parameters. It was done according to three principles: - some of the parameters could be estimated, directly from the data being considered : induction rates $\beta$ (number of local and regional tertiary employment per inhabitant), external demand D (total number of employment. of this activity sector at the end of the period), growing rates $\epsilon$ and $\eta$ for every employment and resident category.

- other parameters could be induced from other data : the proportion of blue collars and white collars working in each of the four economic activities was determined by applying estimations established for french urban population; sensitivity to crowding $\tau$ and $\nu$ were estimated from data about space needed by economic activities on the one hand and mean density by housing-type in Rouen agglomeration on the other hand.

- the remaining parameters had to be estimated, following the relationships between their value and their effects on urban configuration, as established by Allen et al. [2]. 


\section{3 - Calibration}

We started calibration in a very empirical way, changing one value of parameter after another and comparing results obtained by simulation with observed values for each of the six variables in every commune at 1975 .

These "empirical" simulations did not yet lead to a good adjustment. We learned however much through them about sensitivity of the model to different values of parameters. The main issues are the following :

- different sets of values for parameters can generate the same type of spatial structure, for example an almost complete decentralization of industry reported upon one or more peripheral communes, or a highly concentrated distribution of industry or exporting tertiary. These kinds of bifurcations are among the most frequently encountered issues of the model. They are mostly determined by respective values of the $\rho$ parameters (table 3) 。

- Resulting evolution obviously depends on choosen time-lag for simulation. A long period of time furthers apparition of important bifurcations, whereas a shorter period allows a better simulation of smooth increase or decrease as observed in the Rouen commune for industry and exporting tertiary. Sensitivity of the model to parameters values also changes then : for example, variation in the $\rho$ parameters is less determinant upon spatial repartition when time period is short. The model is then more sensitive to $\epsilon$ and $\eta$ parameters.

- from one simulation to another, evolution of spatial repartitions or variation of total quantities of employments and residents are not always directed to the outcome that one would have intuitively expected, by trusting in general significance given to parameters. These counter-intuitive 
Table 3 : Values of parameters and resulting configuration for exporting activities.

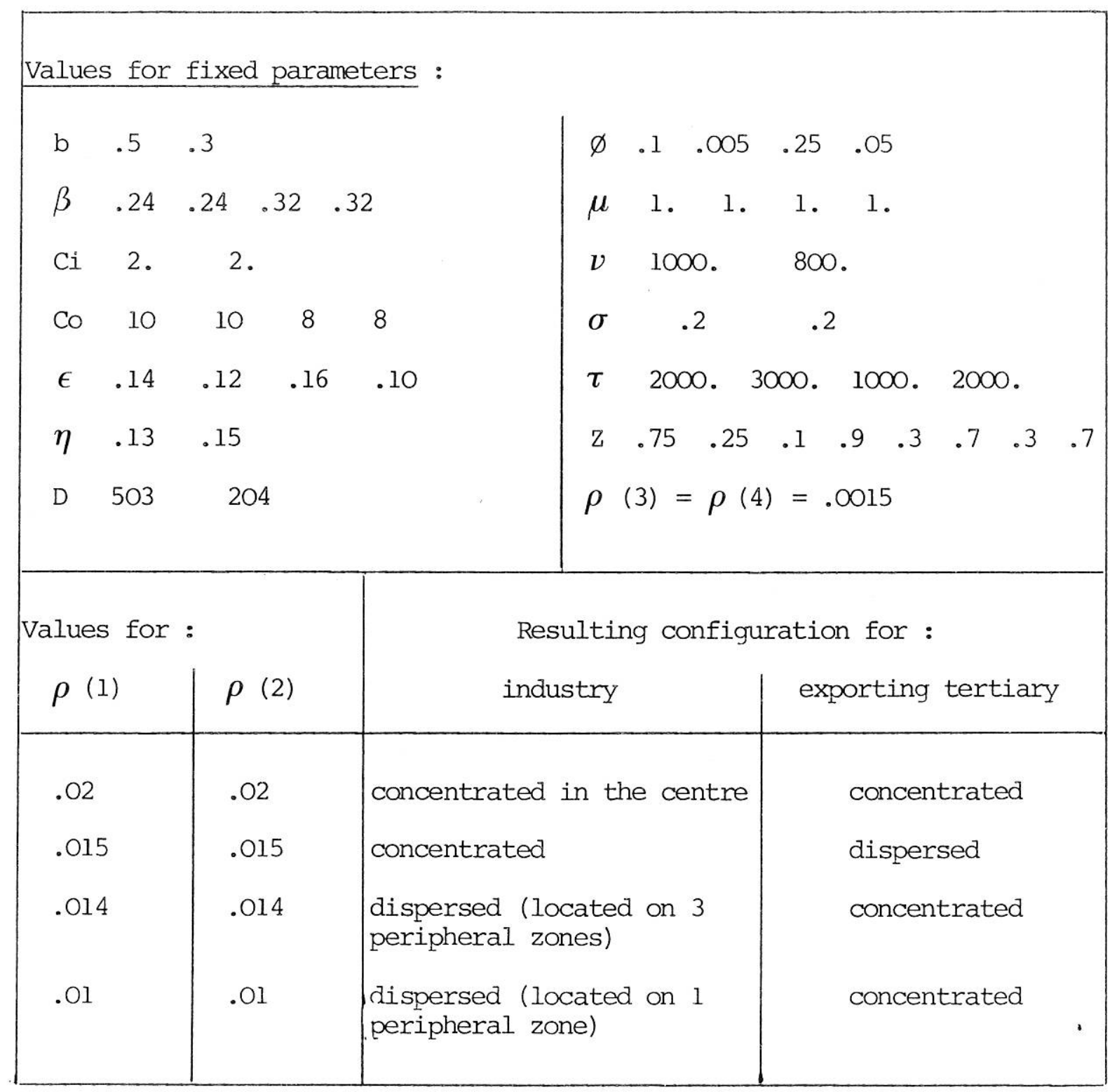


results explain themselves on the whole by non-linearities of the system, butmake rather difficult a progressive "manual" calibration, because of the number of iterative operations that they imply in choosing values for parameters by trial and error.

That is why, after more than a hundred of these tentative simulations, we decided to use an automatic technique for calibration.

To fit the model's parameter to observed data, we used a non-linear least square method. The actual computation was performed with the help of a library subroutine (called MINUIT) designed by nuclear physicists. It is a flexible minimization program incorporating three different minimization procedures which are used successively and in combination. It may in principle handle as many as forty parameters. It appears however preferable for an efficient convergence to subdivide the parameter set into smaller subsets (of four or five parameters each) to be treated successively.

The adjustments that we obtained until now are not good enough to be considered as a "best fit" and to allow calculation of residuals. But we have a serie of interesting results with various configurations according to different values of parameters. In a number of cases, we obtained a good estimation of the total number of employments and residents for the whole. agglomeration but a bad repartition between communes of the center and of the periphery ; in some other cases we obtained an almost perfect adjustement for the center but all variables for the other communes were underestimated. As the model is very sensitive to parameter values, the minimization program can give very different results, depending upon the initial set of values choosen and of the variations which are allowed to them. It 
is therefore necessary to examine in detail each result and to modify the initial configuration of parameters values according to their significance and effect on variables evolution. As these effects are not always the same, depending upon the total configuration being considered, the adjustment procedure can take rather a long time. However, it seems to be consistent from one urban case to another, as preliminary results with data for urban agglomerations of Nantes and Bordeaux already showned.

For each of those agglomerations, main points of disagreement between predicted and actual urban evolution concern rates of variation of employment in exporting activities and their spatial repartition. Two further directions for experiment can then be suggested :

- by replacing final potential demand D by a progressive allocation of newly offered employments, less brutal variations could be generated ; - by modifying accessibility indices of communes, final observed repartition could be better estimated and explanation for these differencies researched afterwards.

We think very plausible to improve the global quality of adjustment between observed and calculated evolution by choosing firsta better set of parameters and using the calibration procedure. That is why we do not propose for the moment a final judgment about the assumed too high complexity of the model ( as in Wilson[5]), or about its ability to reproduce observed evolutions. One problem will nevertheless arise when we will find - if we do so - a "satisfying" fit : is the corresponding set of parameters actually the better one ? and is it unique ? Answers to these questions are, of course, critical if we want to use the INTRA URBAN model as a tool for com- 
paring evolutions of various urban agglomerations - as we hoped by starting this study. It is possible that more insights in theoretical issues of the model are necessary before trying applications - but on the reverse, only applications can reveal practical utility of the model. What is more ungently needed is a serious improvement in the general methodology required for applications of such complex dynamic models to real-world situations.

REFERENCES

[1] ALLEN, P.M., SANGLIER, M., 1978 : Dynamic models of urban growth. Journal of Social and Biological Structures, $n^{\circ} 1$, pp. 265-280, and 1979, $\mathrm{n}^{\circ} 2$, pp. 269-278.

[2] ALLEN, P.M., SANGLIER, M., BOON, F., DENEUBOURG, J.L., DE PALMA, A., 1981 : Models of urban settlement and structure as dynamic self-organizing systems. Washington (D.C.), US Department of Transportation, contract T.S.C. 1640.

[3] PRIGOGINE, I., STENGERS, I., 1979 : La nouvelle alliance. Paris, Gallimard.

[4] ALUEN, P.M., SANGLIER, M., 1979 : A dynamic model of growth in a central place system. Geographical Analysis, vol. 1l, $\mathrm{n}^{\circ} 3$, pp. 256-272.

[5] WIISON, A.G., 1981 : Catastrophe theory and bifurcation : Applications to Urban and Regional Systems. Iondon, Croom Helm. 